\title{
A CASCATA DOS FOSFOINOSITÍDEOS
}

Mauro Vieira de Almeida*, Adilson David da Silva, Marcus Vinícius Nora de Souza e Aloísio Antônio Alves Benício Departamento de Química, ICE, Universidade Federal de Juiz de Fora, Campus Martelo, 36038-330 Juiz de Fora - MG

Recebido em 30/1/02; aceito em 24/6/02

THE PHOSPHOINOSITIDE CASCATE. Inositol is a polyalcohol required for the proper formation of cell membranes. In the body, its plays an important role in the transmission of nerve impulses, its also helps in the transporting of fats within the body. In mammals, inositol exists as phosphorylated derivatives, various phosphoinositides, and in its free form. Agonist stimulated hydrolysis of phosphatidylinositol 4,5-bisphosphate $\left[\mathrm{PI}(4,5) P_{2}\right]$ is the first step in the transmembrane signalling mechanism when cells respond to external stimuli. Under control of activated phospholipase C (PLC) via G-protein, two second messengers Dmyo-inositol 1,4,5-triphosphate $\left[\operatorname{Ins}(1,4,5) P_{3}\right]$ and diacylglycerol are released into the cell. From $\operatorname{Ins}(1,4,5) P_{3}$, enzymatic process under phosphatases or kinases control affords subsequent inositol phosphate metabolites. During the last decade the synthesis of modified inositol phosphate derivatives has been strongly investigated. This paper reviews principal aspects about synthesis and biological functions of these biomolecules.

Keywords: second messengers; phosphoinositides; inositol phosphate.

\section{INTRODUÇÃO À QUÍMICA DOS INOSITÓIS}

O inositol é um poliálcool cíclico contendo um anel de seis átomos de carbono e seis grupos $\mathrm{OH}$ (cicloexanopoliol), sendo um importante constituinte celular, estando envolvido em diferentes processos bioquímicos. Em mamíferos o inositol existe principalmente sob a forma de derivados fosforilados, os quais participam da comunicação celular.

\section{Nomenclatura dos inositóis}

Os inositóis podem ser arranjados em nove estereoisômeros: scilo, mio, neo, epi, D e L quiro, cis, тисо e allo (Figura 1).
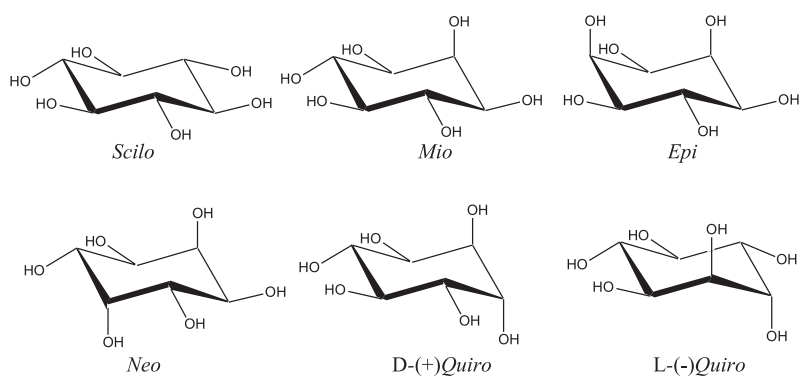

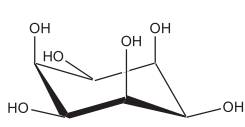

Cis

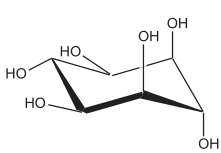

Muco

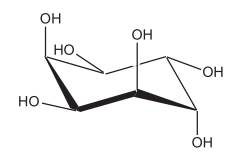

Allo
Figura 1. Estereoisômeros do inositol

Entre os isômeros mostrados na Figura 1, o mio-inositol é o mais abundante na natureza, sendo produzido a partir da glicose. De acor-

*e-mail: mvieira@quimica.ufjf.br do com a nomenclatura oficial, o único grupo hidroxila axial do mioinositol (I) ocupa a posição C-2 na estrutura (Figura 2). A fosforilação em O-1 do mio-inositol leva ao 1L-mio-inositol 1-fosfato (II), no entanto, a fosforilação na posição O-3, altera a numeração dos átomos de carbono, invertendo C-1 com C-3, levando ao 1D-mio-inositol 1-fosfato (III).

O mio-inositol é um composto meso, visto que apresenta um plano de simetria passando pelos átomos C-2 e C-5 (Figura 2). Toda mono-substituição nas posições 1, 3, 4 ou 6 gera, portanto, um racemato.
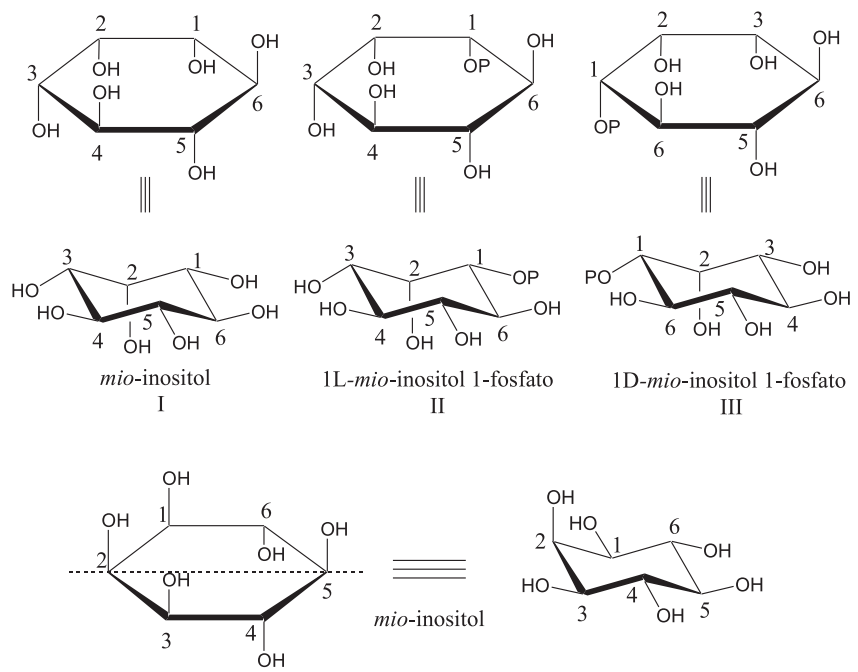

Figura 2. Nomenclatura e plano de simetria do mio-inositol

\section{O símbolo Ins}

O símbolo Ins é utilizado para o mio-inositol com configuração 1D. Caso seja de configuração L, esta deve ser previamente mencionada. A terminação $\mathrm{P}_{x}$, em itálico, indica o número de fosforilações presentes no inositol. 


\section{Exemplo:}

Ins $(1,4,5) P_{3}$ (1D-mio-inositol 1,4,5-trifosfato)

Ins $(1,3,4,5) P_{4}$ (1D-mio-inositol 1,3,4,5-tetraquisfosfato)

Ins $(3,4) P_{2}$ (1D-mio-inositol 3,4-difosfato)

\section{A CASCATA DE FOSFOINOSITÍDEOS}

As comunicações nos organismos superiores são necessárias para o controle do desenvolvimento das células, de suas organizações em tecidos e órgãos, de seus crescimentos e de suas multiplicações, necessárias também à coordenação de suas atividades. Em um sistema de comunicação (Figura 3), um primeiro sinal chamado "primeiromensageiro"' (hormônio, fator de crescimento, etc.) é secretado e circula no meio extracelular. O primeiro mensageiro $(\nabla)$ é captado na superfície da célula por receptores $(\mathrm{R})$ que lhe são específicos. A ocupação dos receptores, inseridos na membrana celular, dá início a eventos complexos na membrana plasmática e no interior da célula.

Um tipo de receptor que apresenta grande importância utiliza uma classe de proteínas chamadas proteínas G. Estas estão ligadas às guaninas sendo acopladas aos canais iônicos, ou a outras enzimas, controlando a liberação de novos mensageiros intracelulares ${ }^{2}$. Estas moléculas biológicas conhecidas com o nome de "segundo-mensageiros" (mensageiros secundários) constituem a última ligação da cadeia de comunicação intracelular antes da resposta fisiológica sendo ponto de grande interesse para a compreensão dos mecanismos de transdução do sinal (Figura 3).

\section{MEIO EXTRACELULAR}

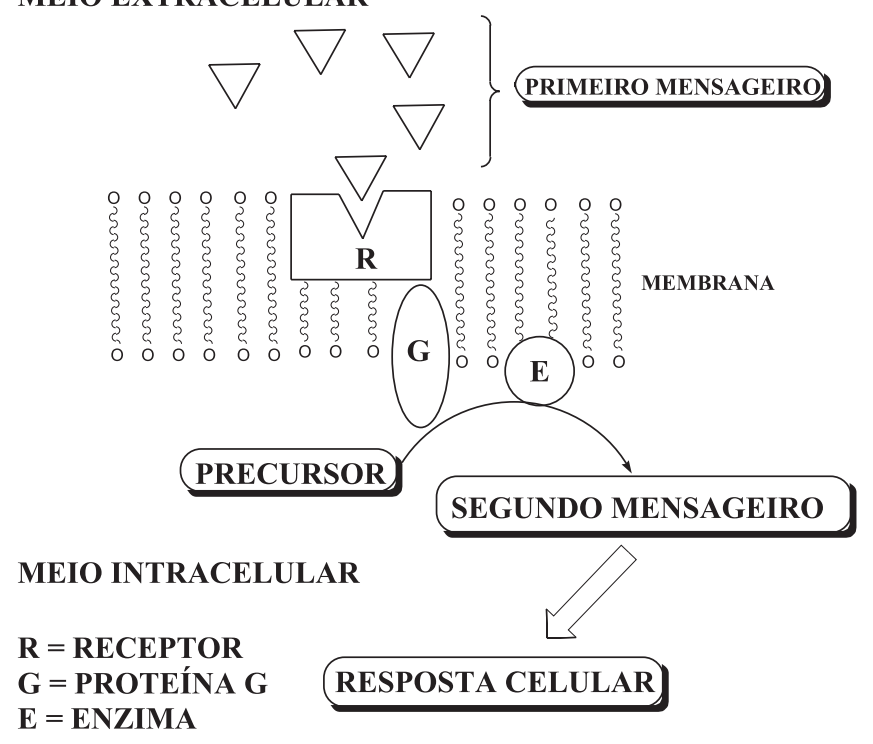

Figura 3. Mecanismo de transdução celular

Em 1975, Michell ${ }^{3}$ propôs uma nova via de transdução de sinal, a qual foi demonstrada por Berridge e colaboradores ${ }^{4}$ em 1983. Porém, somente nos últimos anos surgiu uma idéia compatível com o desempenho fisiológico da cascata de fosfoinositídeos ${ }^{5,6}$.

A partir de uma estimulação induzida por um agonista, ao nível do receptor na membrana, uma proteína $\mathrm{G}$ ativa a fosfolipase C (PLC), a qual catalisa a hidrólise da ligação fosfodiéster do $\operatorname{PI}(4,5) P_{2}$, liberando no meio intracelular dois novos segundos mensageiros: o mioinositol 1,4,5-trifosfato $\left[\operatorname{Ins}(1,4,5) P_{3}\right.$ ] e o $s n$-1,2-diacilglicerol (Figura 4). Este evento dispara uma série de reações seqüenciais denominada cascata de fosfoinositídeos (Figura 5).
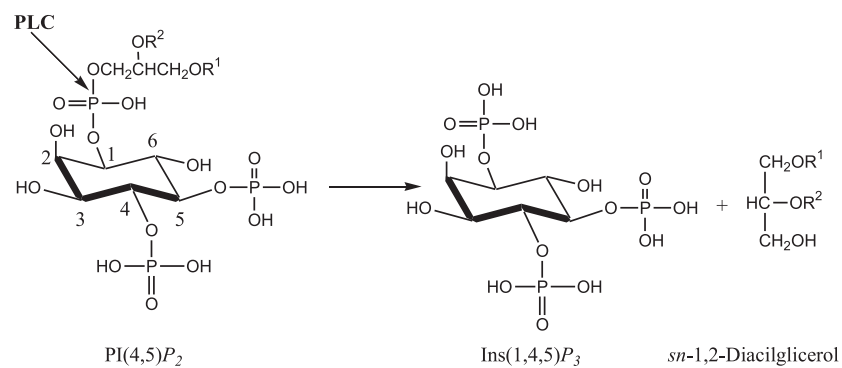

$\mathrm{R}^{1}$ e $\mathrm{R}^{2}=$ Grupos acilas oriundos de ácidos graxos, como por exemplo ácido esteárico e araquidônico

Figura 4. Hidrólise da ligação do $\mathrm{PI}(4,5) \mathrm{P}_{2}$

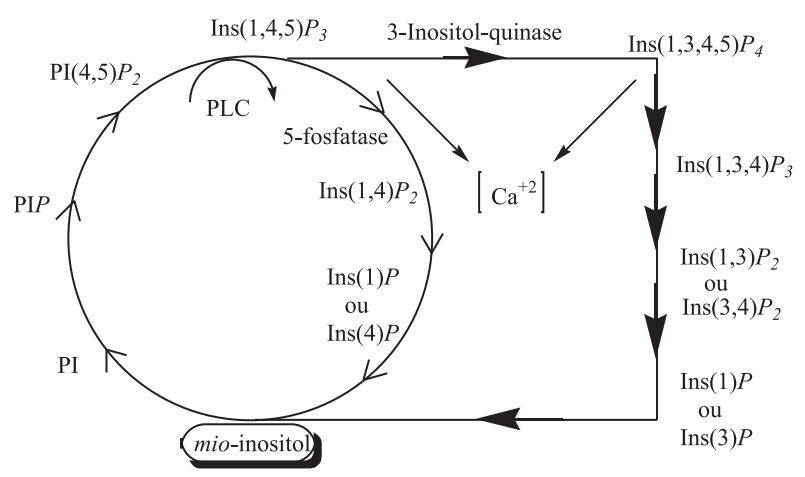

Figura 5. Cascata de fosfoinositídeos

O controle da hidrólise do $\operatorname{PI}(4,5) P_{2}$ é agora reconhecido por ser um dos mecanismos fundamentais na comunicação intercelular. Um grande número de neurotransmissores, hormônios, etc. utilizam este mecanismo de transdução/amplificação para provocar as respostas celulares.

O Ins $(1,4,5) P_{3}$, hidrossolúvel, liga-se a um receptor intracelular específico e mobiliza o $\mathrm{Ca}^{2+}$ presente no retículo endoplasmático de um grande número de sistemas celulares diferentes. Existe um certo número de substâncias que podem bloquear estes receptores e o mais poderoso já identificado é a heparina ${ }^{7}$. O Ins $(1,4,5) P_{3}$ é responsável pela regulagem de numerosos processos celulares, como a secreção, o metabolismo, a contração e a proliferação.

O sn-1,2-diacilglicerol fica na membrana plasmática e age ativando a proteína quinase C (PKC). Esta enzima estimula a fosforilação de numerosas proteínas intracelulares ${ }^{8,9}$.

\section{Metabolismo do $\operatorname{Ins}(1,4,5) P_{3}$}

A mobilização do $\mathrm{Ca}^{2+}$ pelo $\operatorname{Ins}(1,4,5) P_{3}$ pode ser interrompida por duas vias metabólicas diferentes (Figura 5). Na primeira a 5fosfatase corta o grupo fosfato em C-5 para conduzir ao mio-inositol 1,4-difosfato [Ins $(1,4) P_{2}$ ]. Duas outras desfosforilações sucessivas conduzem, via o mio-inositol 4-fosfato $[\operatorname{Ins}(4) P]$, ao mio-inositol que, por fosforilações sucessivas, é de novo transformado em $\mathrm{PI}(4,5) P_{2}$.

A segunda alternativa de metabolismo é a fosforilação do Ins $(1,4,5) P_{3}$ em mio-inositol 1,3,4,5-tetrafosfato $\left[\operatorname{Ins}(1,3,4,5) P_{4}\right]$ pela inositol 3-quinase (Figura 5). Este último é rapidamente desfosforilado pela 5-fosfatase em mio-inositol 1,3,4-trifosfato $\left[\operatorname{Ins}(1,3,4) P_{3}\right]$, depois em mio-inositol 1,3 ou 3,4-difosfato e, finalmente, em mio-inositol, via diferentes monofosfatos. $\mathrm{O} \operatorname{Ins}(1,3,4,5) P_{4}$ pode ligar-se fracamente ao sítio de ligação do $\operatorname{Ins}(1,4,5) P_{3}$, mas 
pode ter ação na mobilização do $\mathrm{Ca}^{2+}$ extracelular através da membrana, quando em presença do $\operatorname{Ins}(1,4,5) P_{3}$.

$\mathrm{O}$ Ins $(1,3,4) P_{3}^{10}$ não estimula a captura do $\mathrm{Ca}^{2+}$. Em 1987, Balla e colaboradore ${ }^{11}$ mostraram a existência de uma quinase que fosforila este trifosfato em mio-inositol 1,3,4,6-tetrafosfato $\left[\operatorname{Ins}(1,3,4,6) P_{4}\right]$, depois em mio-inositol 1,3,4,5,6-pentafosfato $\left[\operatorname{Ins}(1,3,4,5,6) P_{5}\right]$. A existência deste último e do inositol hexafosfato (ácido fítico) foi provada em diferentes tecidos de mamíferos ${ }^{12}$.

\section{Discussão sobre a cascata de fosfoinositídeos}

Apesar de numerosos estudos realizados sobre o papel do Ins $(1,4,5) P_{3}$ e do $s n$-1,2-diacilglicerol como segundo-mensageiros no modo de transdução utilizado na cascata dos fosfoinositídeos, nenhum mecanismo de ação ou uma relação estrutura-atividade pode ser proposto sem ambigüidade.

Sabe-se que o $\operatorname{Ins}(1,4,5) P_{3}$ é um mediador na liberação do $\mathrm{Ca}^{2+}$ intracelular, do retículo endoplasmático (RE) para o citoplasma. Ele ativa um receptor situado na membrana externa do RE e ligado a um canal de cálcio. Esta ativação provoca a abertura deste último e, assim, a liberação do $\mathrm{Ca}^{2+}$ no citoplasma.

Sabemos também que a capacidade dos inositóis fosfatos de provocar a liberação do cálcio depende do número e da posição destes grupos fosfatos na molécula. A presença de grupo fosfato na posição C-1 é indispensável para fazer a ligação com o receptor. Estudos feitos sobre a relação estrutura-atividade indicam que a presença de grupos fosfatos vicinais nas posições 4 e 5 do mio-inositol é essencial para a liberação do $\mathrm{Ca}^{2+}$, como havia previsto Irvine em $1984^{13}$.

Tem-se igualmente evidenciado que a posição 2 no mio-inositol (hidroxila em axial) tem um papel particular, pois esta posição é importante para o reconhecimento dos inositóis fosfatos por diferentes enzimas ${ }^{14}$.

A descoberta recente do fosfatidilinositol 3-fosfato e de um fosfatidilinositol 3,4,5-trifosfato indica que existem outros fosfoinositídeos ainda desconhecidos.

Não se sabe qual é a importância fisiológica da transformação do Ins $(1,4,5) P_{3}$ em diversos compostos fosforilados. Não se sabe se estes compostos têm uma atividade biológica própria ou se são somente metabólitos intermediários.

O mio-inositol 1,2,6-trifosfato $\operatorname{Ins}(1,2,6) P_{3}$ é um produto obtido pela degradação enzimática do ácido fítico (mio-inositol 1,2,3,4,5,6hexafosfato $)^{15}$. Este trifosfato mostrou efeitos farmacológicos importantes em muitas patologias, como por exemplo nas complicações diabéticas secundárias, nas doenças cardiovasculares e nas inflamações crônicas tais como artrite ${ }^{15}$.

\section{PRINCIPAIS METODOLOGIAS DE SÍNTESE DOS METABÓLITOS (E DE SEUS ANÁLOGOS) DA CASCATA DE FOSFOINOSITÍDEOS.}

\section{Principais materiais de partida}

O mio-inositol, acessível e barato, é o material de partida mais utilizado para a síntese de inositóis. Entretanto, muitas outras substâncias têm sido utilizadas, em particular os inositóis quirais naturais isolados a partir de plantas D-pinitol, L-quebrachitol e o ácido (-)quínico e os arenos que, por oxidação microbiana pela Pseudomonas putida, conduzem aos 1,2-diidroxicicloexano-3,5-dieno (Figura 6). Outro material de partida muito utilizado por Gero e colaboradores $^{16-28}$ para a síntese de desoxi-inositóis fosfatos são os monossacarídeos, principalmente D-galactose e D-glicose, conforme exemplificado no Esquema 1 onde a rota sintética utiliza como etapa-chave o rearranjo de Ferrier. Este rearranjo consiste na conversão de um 6-desoxi-5-hexeno-piranosídeo em cicloexanonas quirais pela reação com sais de mercúrio(II) ${ }^{26,29-31}$, permitindo a transformação de um monossacarídeo em uma cicloexanona poliidroxilada, que pode ser reduzida à um cicloexanopoliol (Esquema 2). Devido à impor-

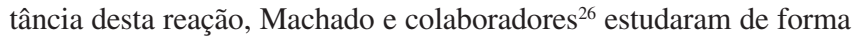
detalhada seu mecanismo, utilizando-se de diversos hexenopiranosídeos e verificando a relação entre a conformação do monossacarídeo de partida e a estereoquímica do produto obtido (alfa ou beta). Segundo estes autores, a explicação estereoeletrônica envolve inicialmente a adição do mercúrio à ligação olefínica fornecendo um intermediário que é hidrolisado gerando 1. A formação deste é seguida pela abertura do anel piranosídico com concomitante perda de metanol fornecendo o ceto-aldeído intermediário 2 que, por sua vez, pode gerar por tautomerismo dois intermediários enólicos 3 e 4. O intermediário 3 ciclizará, de maneira análoga a uma condensação aldólica intramolecular, para formação do isômero beta, enquanto o intermediário $\mathbf{4}$ conduzirá ao isômero alfa, este último normalmente obtido majoritariamente.

A utilização do mio-inositol como material de partida cria um problema ligado a sua geometria, pois este inositol é um composto

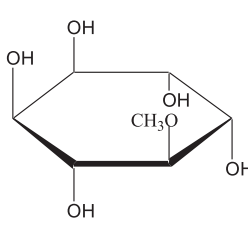

D-Pinitol

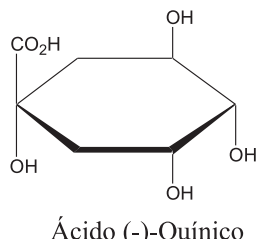

Ácido (-)-Quínico

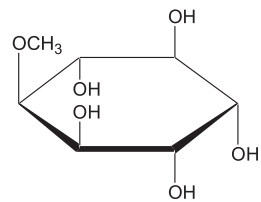

L-Quebrachitol<smiles>[R]c1ccccc1</smiles>

Pseudomonas putida

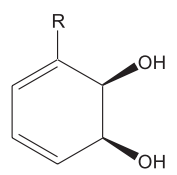

$\mathrm{R}=\mathrm{H}, \mathrm{CH}_{3}, \mathrm{Cl}$, etc

Figura 6. Precursores de mio-inositois fosfatos quirais sintéticos
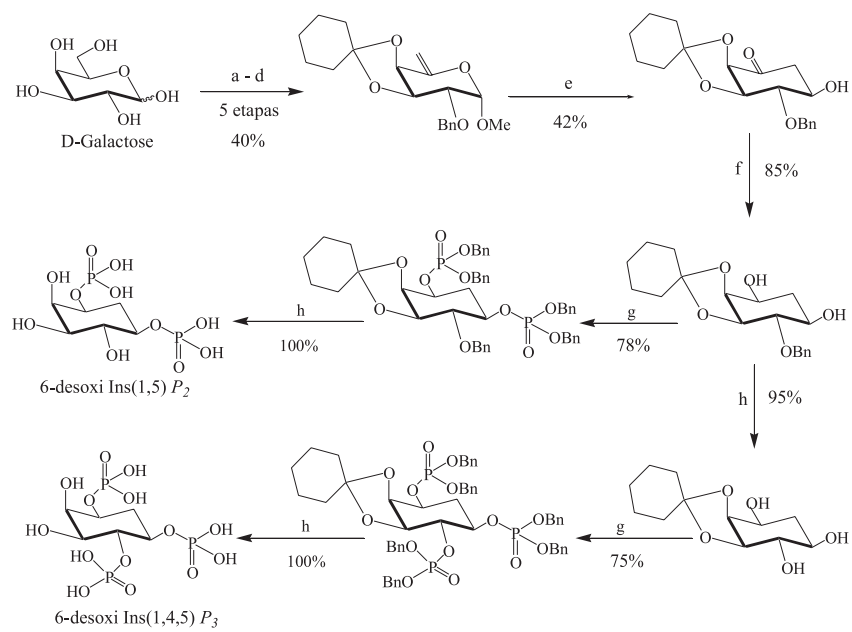

a) Cicloexanona, $\mathrm{H}_{2} \mathrm{SO}_{4}$, DMF; b) $\mathrm{CBr}_{4}, \mathrm{PPh}_{3}$, Pir.; c) $\mathrm{NaH}, \mathrm{DMF}, \mathrm{BnBr}$; d) $\mathrm{NaH}$, DMF; e) $\mathrm{HgCl}_{2}$, acetona- $\mathrm{H}_{2} \mathrm{O}$; f) $\left.\mathrm{NaBH}_{4}, \mathrm{THF} ; \mathrm{g}\right)(\mathrm{BnO})_{2} \mathrm{PNiPr}_{2}, 1 \mathrm{H}$ - tetrazol, $\mathrm{CH}_{2} \mathrm{Cl}_{2}$, então tBuOOH; h) $\mathrm{H}_{2}$, $\mathrm{Pd} / \mathrm{C}$

Esquema 1. Síntese do 6-desoxi Ins $(1,4,5) \mathrm{P}_{3}$ a partir da D-galactose via rearranjo de Ferrier 

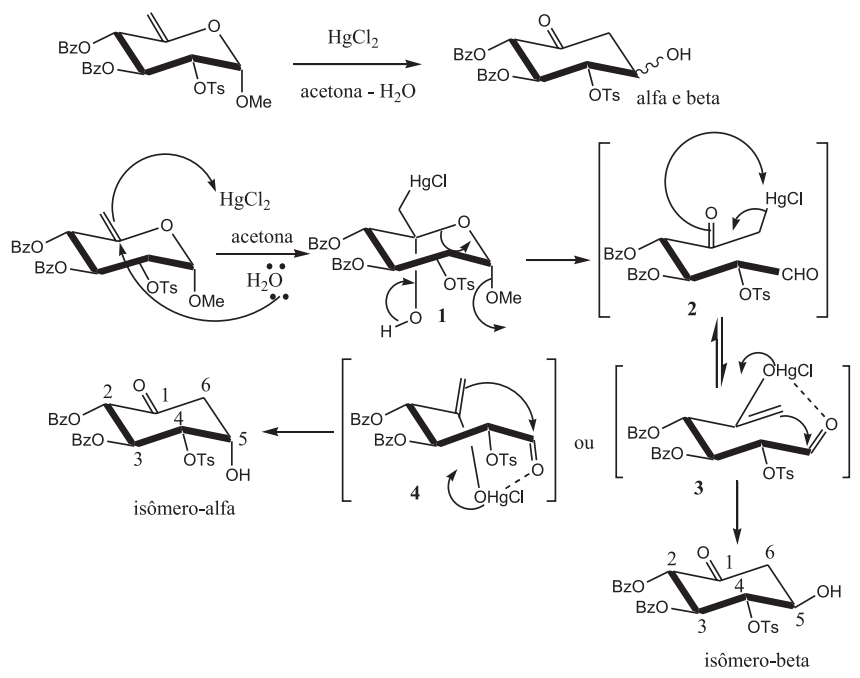

Esquema 2. Mecanismo do rearranjo de Ferrier

meso, visto que apresenta um plano de simetria passando pelos átomos C-2 e C-5, conforme discutido anteriormente (ver Figura 2).

A síntese dos diferentes inositóis fosfatos compreende quatro etapas principais:

- a resolução dos inositóis racêmicos;

- a obtenção de inositóis convenientemente protegidos (regiosseletividade nas reações);

- a fosforilação eficaz dos grupos $\mathrm{OH}$, evitando a formação de fosfatos cíclicos ou a migração dos grupamentos fosfatos;

- a desproteção dos fosfatos e das hidroxilas em condições suaves para facilitar o isolamento e a purificação dos produtos, evitando assim a formação de compostos secundários indesejáveis.

\section{RESOLUÇÃO DOS RACEMATOS}

Para a resolução dos derivados racêmicos do mio-inositol, um dos métodos é a transformação destes em ésteres diastereoisoméricos, seguida da separação por cromatografia ou cristalização seletiva de um dos diastereoisômeros. Os três ésteres mais utilizados são: os mentóxi acetatos ${ }^{32}$, os canfonatos ${ }^{33}$ e os ortoésteres de D-manose ou D-glicose ${ }^{34}$ (Figura 7).

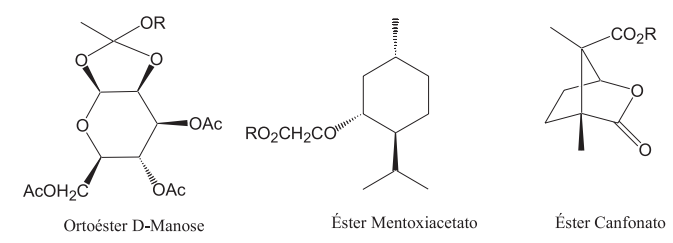

Figura 7.

Um outro método ${ }^{35}$ utiliza como auxiliar quiral a D-cânfora, e leva à obtenção, por resolução cinética, de $65-70 \%$ do derivado cristalino 2,3-O-cetálico D-mio-inositol. Este diastereoisômero é obtido por simples filtração da mistura reacional (Esquema 3).

\section{Grupos protetores e métodos de fosforilação}

Os grupos protetores mais usados para as hidroxilas são os grupos benzilas, benzoílas, cetais (cicloexilidenos e isopropilidenos), alilas, ortoformatos, sililas, acetatos, metoximetilas, $p$-metoxibenzilas

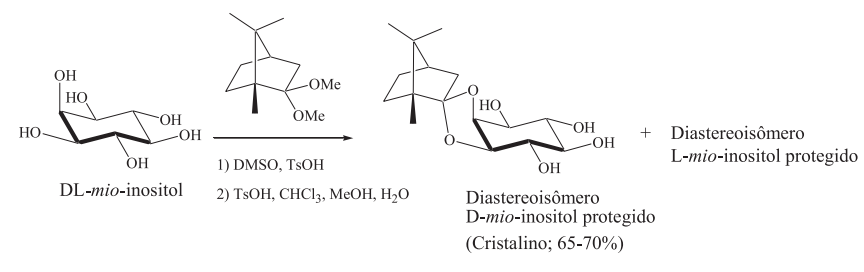

Esquema 3. Resolução cinética do mio-inositol

e pivaloílas. Os grupos fosfatos são geralmente protegidos sob forma de benzila, fenila ou cianoetila.

Diversos métodos de fosforilações são descritos e a escolha depende dos grupos protetores que existem no material de partida e do composto que se deseja obter. Um método muito utilizado e que assegura uma fosforilação eficaz tem como intermediário um fosforamidito P(III), seguido de oxidação in situ a fósforo (V) (Esquema 4). As condições requeridas para este método são suaves e permitem a obtenção de diferentes análogos ao nível dos grupos fosfato, como por exemplo os fosforotioatos.

Uma segunda alternativa implica na reação do ânion alcóxido, gerado pela ação do $n$-butil lítio, com o tetrabenzilpirofosfato (Esquema 4). Em certos casos, a fosforilação por este método não assegura um bom rendimento e as condições básicas podem ser inconvenientes.

$\mathrm{O} \mathrm{PCl}_{3}$ pode também ser utilizado para a fosforilação de um $\mathrm{OH}$ isolado, sobretudo se este grupo se situa na posição C-2, mas a formação de fosfatos cíclicos e outros derivados não desejados impede a utilização deste reativo para fosforilação de hidroxilas vicinais. Um outro método utiliza o cloreto do ácido di- $n$-alquil fosfórico, porém o resultado depende muito da posição da hidroxila a ser fosforilada (Esquema 4).

Nas duas primeiras opções de métodos de fosforilação, a presença de ésteres benzílicos sobre os fosfatos impede sua migração e permite a desproteção dos fosfatos livres em condições muito suaves (hidrogenólise). O produto é obtido diretamente após filtração e evaporação a seco.
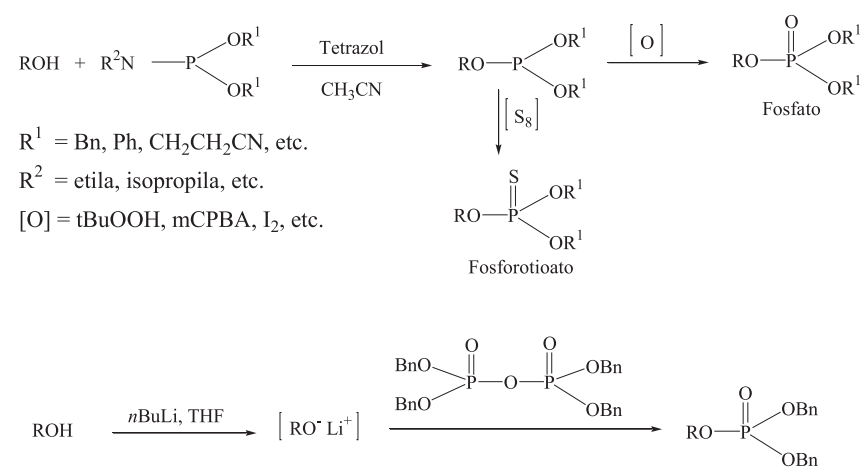

$\mathrm{ROH}+\mathrm{PCl}_{3} \frac{\text { Tolueno }}{\mathrm{SO}_{2} \mathrm{Cl}_{2}} \mathrm{Cl}-\left\|_{\mathrm{P}}^{\mathrm{O}}-\mathrm{OR} \underset{\mathrm{OR}}{\stackrel{\mathrm{R}^{1} \mathrm{OH}}{\text { Base }}} \mathrm{R}^{1} \mathrm{O}-\right\|_{\mathrm{P}}^{\mathrm{O}}>_{\mathrm{OR}}$

Esquema 4. Métodos de fosforilação envolvendo o fosforamidito, pirofosfato ou o cloreto do ácido di-n-alquil fosfórico

\section{EXEMPLOS DE SÍNTESE DOS METABÓLITOS (E DE SEUS ANÁLOGOS) DA CASCATA DE FOSFOINOSITÍDEOS}

Discutiremos, a seguir, exemplos de métodos de preparação do Ins $(1,4,5) P_{3}$ e de alguns de seus análogos, utilizando diferentes materiais de partida, grupos protetores e métodos de fosforilação. 
Uma síntese versátil e enantioespecífica do $\operatorname{Ins}(1,4,5) P_{3}$ em 15 etapas (rendimento global 6,2\%) a partir do ácido (-)-quínico comercial foi realizada por Falck e Yadagiri ${ }^{36}$ (Esquema 5).

O ácido (-)-quínico 5 foi inicialmente convertido no éster 6 em 4 etapas de acordo com procedimento descrito previamente na literatura $^{36}$. A proteção da hidroxila com o grupo trimetilsilil etoximetila (SEM), seguida da redução da função éster com hidreto de diisobutilalumínio (DIBAL-H) e selenilação do álcool resultante forneceu o produto 7. Rearranjo do selenóxido alílico 7 e posterior benzilação gerou o composto $\mathbf{8}$. A transformação de $\mathbf{8}$ no enol sililado 9 foi realizada por ozonólise e posterior tratamento com excesso de triflato de tert-butildimetilsilila. A hidroboração de $\mathbf{9}$ a partir da face $\beta$, menos impedida, seguida por oxidação alcalina com peróxido de hidrogênio e posterior desililação levou ao triol 10. Fosforilação de 10 usando pirofosfato de tetrabenzila em presença de hidreto de potássio e posterior remoção dos grupos protetores conduziu ao composto desejado $\operatorname{Ins}(1,4,5) P_{3} \mathbf{1 1}$.
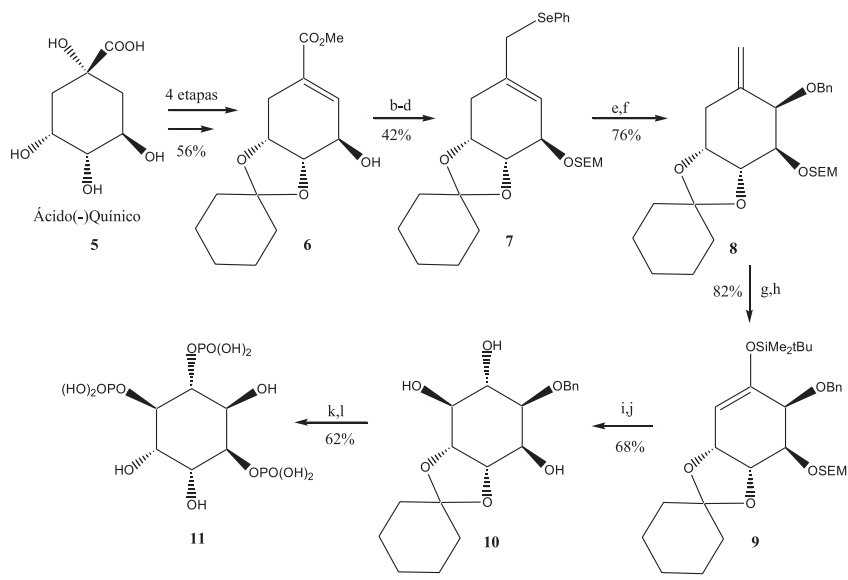

(a) 4 etapas; (b) SEM-Cl, $i$ - $\mathrm{Pr}_{2} \mathrm{NEt}, \mathrm{THF}, 45^{\circ} \mathrm{C}, 12 \mathrm{~h}$; (c) DIBAL-H, $\mathrm{PhCH}_{3},-78^{\circ} \mathrm{C}, \quad 3 \mathrm{~h}$; (d) $\mathrm{N}$-(fenilseleno)ftalimida, $\mathrm{Bu}_{3} \mathrm{P}, \mathrm{THF}, 0^{\circ} \mathrm{C}, 45 \mathrm{~min}$.; (e) $\mathrm{NaIO}_{4}$, tampão $\mathrm{pH}$ 7, 1,4 -dioxano- $\mathrm{H}_{2} \mathrm{O}$; (f) $\mathrm{KH}, \mathrm{BnBr}$, THF, $12 \mathrm{~h}$; (g) $\mathrm{O}_{3}, \mathrm{CH}_{2} \mathrm{Cl}_{2}-\mathrm{MeOH},-78^{\circ} \mathrm{C} ; \mathrm{Me}_{2} \mathrm{~S},-78^{\circ} \mathrm{C}$; (h) t-BuMe $\mathrm{SiOTf}_{2} \mathrm{Et}_{3} \mathrm{~N}$, $\mathrm{CH}_{2} \mathrm{Cl}_{2}, 2 \mathrm{~h}$; (i) $\mathrm{BH}_{3}, \mathrm{THF}, 25^{\circ} \mathrm{C}, 3 \mathrm{~h}$; NaOH, $\mathrm{H}_{2} \mathrm{O}_{2}, 1 \mathrm{~h}$; (j) $\mathrm{n}-\mathrm{Bu}_{4} \mathrm{NF}, \mathrm{HMPA}, 100^{\circ} \mathrm{C}, 1 \mathrm{~h}$; (k) $\mathrm{KH}$, $\left[(\mathrm{BnO})_{2} \mathrm{PO}\right]_{2} \mathrm{O}$, THF, $60{ }^{\circ} \mathrm{C}$; (l) $\mathrm{H}_{2}, 10 \%$ Pd / C, 50 psi, EtOH-AcOH- $\mathrm{H}_{2} \mathrm{O}$

Esquema 5. Síntese do Ins(1,4,5) $\mathrm{P}_{3}$ a partir do ácido (-)-quínico

Kozikowski e colaboradores ${ }^{37}$ realizaram a síntese do $\operatorname{Ins}(1,4,5) P_{3}$ 20, modificado na posição C-3, em 16 etapas (rendimento global $4,8 \%$ ) utilizando como composto de partida o L-quebrachitol 12 (Esquema 6). O L-quebrachitol, um material de partida quiral e bastante versátil, pode ser facilmente obtido a partir do extrato aquoso do látex de seringueiras (Hevea brasiliensis). A etapa-chave desta síntese é a introdução do grupo trifluormetila através da oxidação do grupo hidroxila de 13, segundo as condições de Swern, e posterior tratamento com trifluormetil trimetilsilano em presença de fluoreto de tetrabutilamônio.

Ley e colaboradores ${ }^{38}$ realizaram a síntese de diferentes análogos do Ins $(1,4,5) P_{3}$ a partir do benzeno, via oxidação microbiana pela Pseudomonas putida (Esquema 7).

O 1,2-diol 22 foi convertido no 6-desoxi-6-metil Ins(1,4,5) $P_{3} 26$ em 8 etapas, com um rendimento global de $18 \%$. O 1,2-diol cicloexadieno 22 foi inicialmente convertido, em 4 etapas, no acetonídeo 23. A abertura nucleofílica do epóxido usando o reagente organocúprico $\mathrm{Me}_{2} \mathrm{Cu}(\mathrm{CN}) \mathrm{Li}_{2}$ forneceu o álcool $\mathbf{2 4}$, que por hidrogenólise e posterior reação de fosforilação com o n-butil lítio/ tetrabenzilpirofosfato forneceu o derivado trifosfato $\mathbf{2 5}$. Total desproteção, segundo o procedimento de Meek (TMSBr), conduziu ao produto desejado $\mathbf{2 6}$.
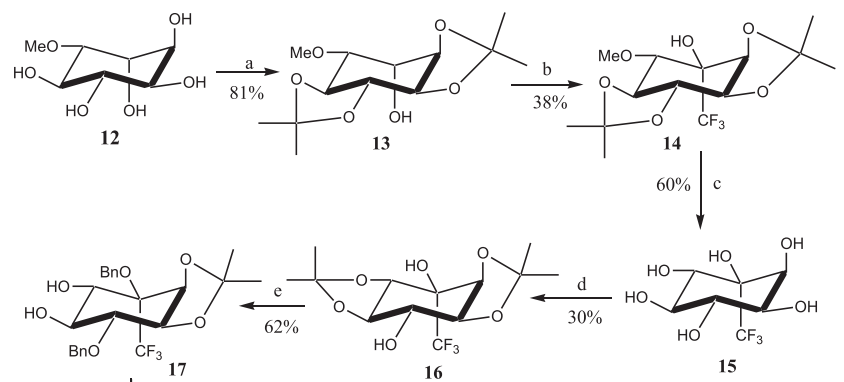

$$
15 \%\lfloor\mathrm{f}
$$

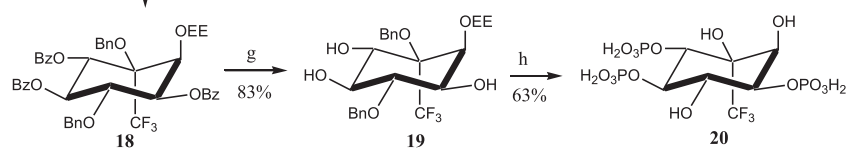

a) $\mathrm{H}_{2} \mathrm{C}=\mathrm{C}(\mathrm{OMe}) \mathrm{Me}$, ácido canforssulfônico (CSA), DMF, $60^{\circ} \mathrm{C}, 4 \mathrm{~h}$; (b) (i) oxidação de Swern $(\mathrm{COCl})_{2}, \mathrm{Me}_{2} \mathrm{SO}, \mathrm{CH}_{2} \mathrm{Cl}_{2}$; então $\mathrm{Et}_{3} \mathrm{~N},-78^{\circ} \mathrm{C}$ a $-30^{\circ} \mathrm{C}, 1 \mathrm{~h}$; (ii) $\mathrm{TMS}^{-\mathrm{CF}_{3}}, \mathrm{Bu}_{4} \mathrm{NF}, \mathrm{THF}, 1 \mathrm{~h} ;$ (c) $\mathrm{BBr}_{3}$, $\mathrm{CH}_{2} \mathrm{Cl}_{2},-43^{\circ} \mathrm{C}$ a $0{ }^{\circ} \mathrm{C}, 12 \mathrm{~h}$; (d) idem (a); (e) (i) $\mathrm{NaH}, \mathrm{BnBr}, \mathrm{DMF}, 0^{\circ} \mathrm{C}, 1 \mathrm{~h}$; (ii) $\mathrm{AcCl}$ (catalítico), $\mathrm{MeOH}-\mathrm{CH}_{2} \mathrm{Cl}_{2}(1: 2), 1 \mathrm{~h}$; f) (i) $\mathrm{PhCOCl}$, pir., DMAP, $25^{\circ} \mathrm{C}, 16 \mathrm{~h}$; (ii) $\mathrm{HCl}$ conc. (catalítico), MeOH - THF 4:1, refluxo, $2 \mathrm{~h}$; (iii) $\mathrm{PhCOCl}$, pir., DMAP, $25^{\circ} \mathrm{C}, 16 \mathrm{~h}$; (iv) $\mathrm{H}_{2} \mathrm{C}=\mathrm{CHOEt}$, $p$-toluenossulfonato de piridínio (catalítico), $\left.\mathrm{CH}_{2} \mathrm{Cl}_{2}, 36 \mathrm{~h} ; \mathrm{g}\right) \mathrm{K}_{2} \mathrm{CO}_{3}, \mathrm{MeOH}, 25^{\circ} \mathrm{C}, 1 \mathrm{~h}$; h) (i) $\mathrm{NaH}$ pirofosfato de tetrabenzila, DMF, $0^{\circ} \mathrm{C}$ a $25^{\circ} \mathrm{C}, 16 \mathrm{~h}$; (ii) $\mathrm{H}_{2}(50 \mathrm{psi}), 10 \% \mathrm{Pd} / \mathrm{C}$; EtOH

Esquema 6. Síntese do Ins $(1,4,5) \mathrm{P}_{3}$ a partir do L-quebrachitol

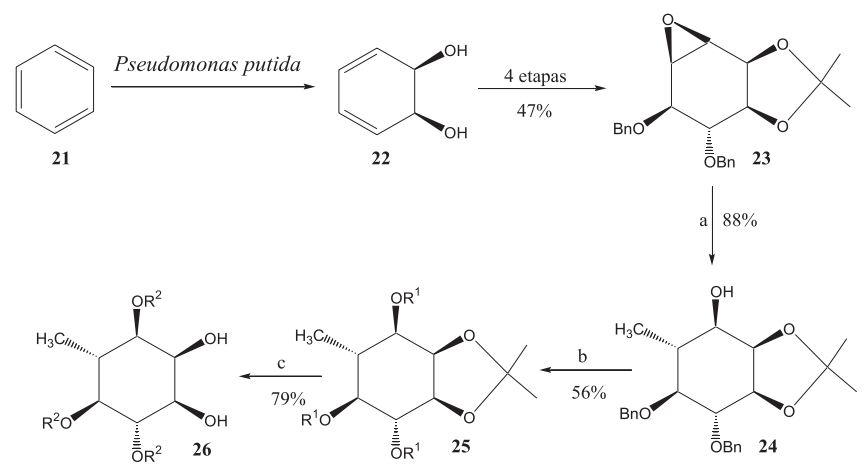

(a) $\mathrm{Me}_{2} \mathrm{Cu}(\mathrm{CN}) \mathrm{Li}_{2}, \mathrm{THF},-30^{\circ} \mathrm{C}, 22 \mathrm{~h}$; (b) $\mathrm{H}_{2}, 10 \% \mathrm{Pd} \mathrm{C}, \mathrm{EtOH}, 16 \mathrm{~h}$; (ii) $n$ - $\mathrm{BuLi}, i-\mathrm{Pr}_{2} \mathrm{NH}, \mathrm{THF}$; (iii) $\left[(\mathrm{BnO})_{2} \mathrm{PO}\right]_{2} \mathrm{O}$; c) (i) $\mathrm{TMSBr} \mathrm{CH}_{2} \mathrm{Cl}_{2}$, a., 65 min.; (ii) $\mathrm{H}_{2} \mathrm{O}, 80 \mathrm{~min}$. $\mathrm{R}^{1}=\mathrm{P}(\mathrm{O})(\mathrm{OBn})_{2} ; \mathrm{R}^{2}=\mathrm{P}(\mathrm{O})(\mathrm{OH})_{2}$

Esquema 7. Síntese de análogos do Ins $(1,4,5) \mathrm{P}_{3}$ a partir do benzeno

Spiers e colaboradores ${ }^{39}$ sintetizaram, a partir do mio-inositol, o Ins $(1,2,3) P_{3}$, o qual apresentou uma alta afinidade na complexação com íons $\mathrm{Fe}^{+3}$, apresentando também propriedades antioxidantes (Esquema 8).

O diacetonídeo 28, preparado pela reação do mio-inositol com o 1,1-dietoxicicloexano, foi convertido no $\operatorname{Ins}(1,2,3) P_{3}$ em 7 etapas, com um rendimento global de 9,2\%. A conversão de $\mathbf{2 8}$ em $\mathbf{2 9}$ foi realizada por remoção seletiva do anel trans-cicloexilideno. O tetrol 29 foi regiosseletivamente sililado na posição 1 , seguido de benzoilação fornecendo o composto tribenzoilado 30. Remoção dos grupos cis-cicloexilideno e silila, pela ação do ácido trifluoracético aquoso, conduziu ao intermediário 31. O 1,2,3-triol 31 foi fosforilado pelo método do fosforamidito ( $\mathrm{N}, \mathrm{N}^{\prime}$-diisopropilfosforamidito de dibenzila/ $m$-CPBA) fornecendo 32, que foi totalmente desprotegido, conduzindo ao composto final $\operatorname{Ins}(1,2,3) P_{3} \mathbf{3 3}$.

Gero e colaboradores sintetizaram diferentes análogos dos inositóis fosfatos a partir do intermediário D-6-desoxi-mio-inositol 

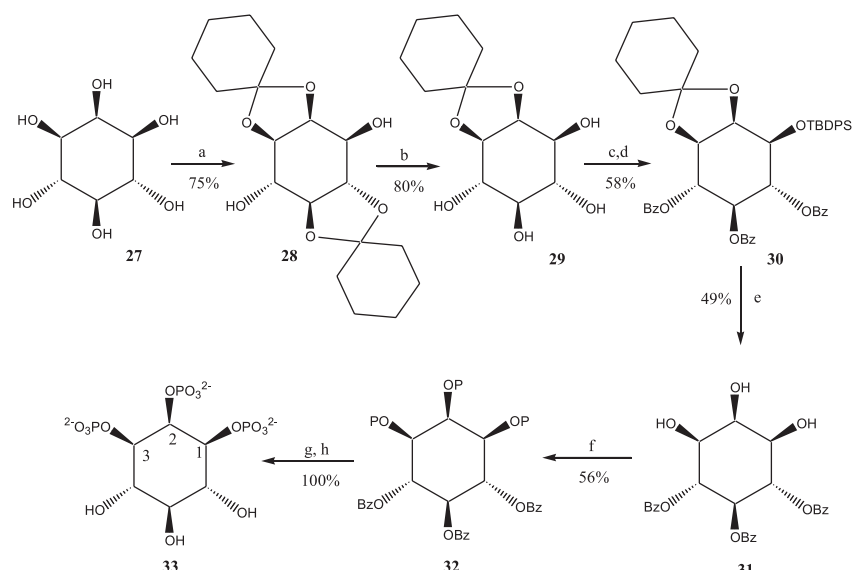

a) Dietoxicicloexano, $\mathrm{TsOH}, \mathrm{MeOH}$; b) $p$-TsOH, tolueno, hexano, EtOH; c) $\mathrm{TBDSCl}$, imidazol, pir. d) $\mathrm{BzCl}$, DMAP, pir.; e) $\mathrm{CF}_{3} \mathrm{CO}_{2} \mathrm{H}$, aquoso; f) $\left(\mathrm{BnO}_{2} \mathrm{PN} i \mathrm{Pr}_{2}, 1 \mathrm{H}\right.$ - tetrazol, $\mathrm{CH}_{2} \mathrm{Cl}_{2}$, então $m$-CPBA g) $\mathrm{H}_{2}, \mathrm{Pd} / \mathrm{C}(10 \%)$, EtOH, t.a. 18 h; h) $\mathrm{NaOH}(0.5 \mathrm{M})$

$\mathrm{P}=(\mathrm{BnO})_{2} \mathrm{P}(\mathrm{O})$

Esquema 8. Síntese do Ins(1,2,3) $\mathrm{P}_{3}$ a partir do mio-inositol

convenientemente protegido $34^{16-28}$. Como exemplo citaremos a síntese do D-2,6-didesoxi-2,2-difluor-Ins $(1,4,5) \mathrm{P}_{3} 42$ (rendimento global $22,4 \%$, Esquema 9) $)^{19,21,25}$.

O material de partida, opticamente ativo, diol 34, foi sintetizado a partir da D-galactose conforme descrito no Esquema 1. O composto $\mathbf{3 4}$ foi benzilado fornecendo o intermediário tribenzilado 35 . Hidrólise ácida do grupo cicloexilideno seguida de benzoilação regiosseletiva forneceu o derivado 37 . Este, por sua vez, foi oxidado pela reação com TPAP/NMO gerando a inosose 38, que foi tratada com DAST levando à formação do derivado 2,2-difluorado 39. Hidrogenólise do grupamento benzila seguida da fosforilação pelo método do fosforamidito e, finalmente, corte do grupo benzoíla em condições básicas, forneceu o composto final desejado $\mathbf{4 2}$.

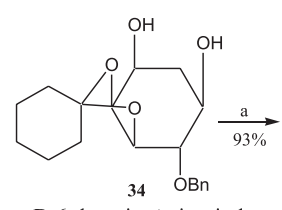
protegido
D-6-desoxi-mio-inositol
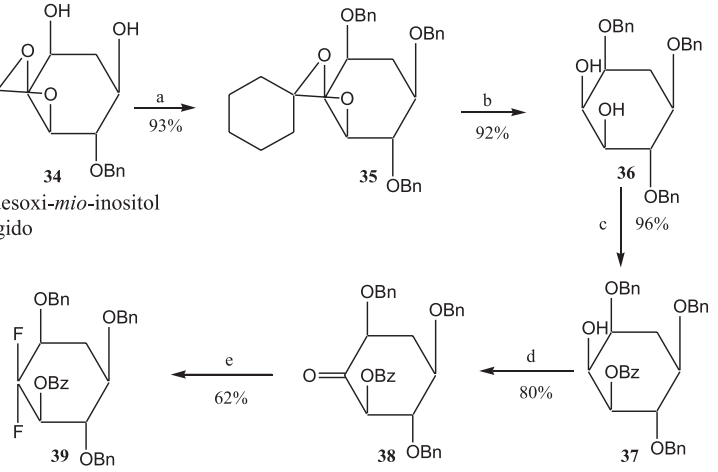

$$
\text { f } \downarrow 100 \%
$$
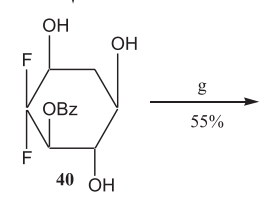
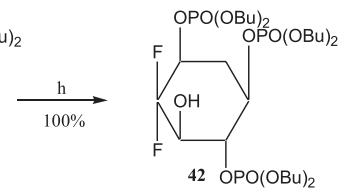

(a) $\mathrm{BnBr}, \mathrm{NaH}$, DMF; (b) $\mathrm{HCl}, \mathrm{MeOH}$; (c) $\mathrm{BzCl}$, pir.; (d) TPAP, NMO, $\mathrm{CH}_{2} \mathrm{Cl}_{2}$ (e) DAST, $\mathrm{CH}_{2} \mathrm{Cl}_{2}$; (f) $\mathrm{H}_{2}$, Pd/C, $10 \%$, 3psi, EtOH; (g) (i) $i-\mathrm{Pr}_{2} \mathrm{NP}(\mathrm{OBu})_{2} 1 H$-tetrazol, (ii) $t$ - $\mathrm{BuOOH}$; (h) $\mathrm{BuONa}^{+}, \mathrm{BuOH}-\mathrm{CH}_{2} \mathrm{Cl}_{2}$

Esquema 9. Síntese de inositois fosfatos difluorados

\section{ATIVIDADES BIOLÓGICAS}

Dentre os análogos mais pesquisados, os do mio-inositol 1,4,5trifosfato constituem o objetivo principal dos pesquisadores. Em 1987, Potter e Gigg ${ }^{40}$ sintetizaram o mio-inositol 1,4,5-tritiofosfato. Este análogo fosforotioato é um agonista do $\operatorname{Ins}(1,4,5) P_{3}$ pela mobilização do $\mathrm{Ca}^{2+}$ intracelular em uma grande variedade de sistemas ${ }^{41-43}$. Este derivado é resistente à desfosforilação catalisada pela 5 -fosfatase ${ }^{44,45}$, sendo o mais poderoso inibidor já descrito desta enzima ${ }^{46}$. Outros derivados halogenados e os mono e ditiofosfatos foram sintetizados com o objetivo de melhorar a interação com as enzimas da cascata, mas eles não trouxeram vantagens suplementares.

Muitos outros análogos do $\operatorname{Ins}(1,4,5) P_{3}$ e certos metabólitos da cascata de fosfoinositídeos foram sintetizados. Como exemplo podemos citar os derivados halogenados, os mono-, di- e tri-desoxi mio-inositol fosfatos. Os únicos que apresentam atividade considerável de agonista são os análogos do $\operatorname{Ins}(1,4,5) P_{3}$ modificados nas posições 2 e 3 (desoxi e desoxifluorados) e o análogo 3,5,6-tridesoxi do mio-inositol 1-monofosfato, que foi identificado como um poderoso inibidor da inositol monofosfatase ${ }^{47}$.

Estudos têm demonstrado que o mio-inositol, o isômero mais abundante no cérebro, e os inositóis fosfatos podem representar uma via para o tratamento das doenças Maníaco-Depressivas, de Alzheimer e Síndrome de Down ${ }^{48-50}$. Além disso, os análogos de D-3-desoximio-inositóis são inibidores do crescimento de células v-sis-transformada NIH 3T3. Os compostos mais ativos são D-3-desoxi-3-cloro-mio-inositol, D-3-desoxi-3-azido-mio-inositol e D-3-desoxi-3flúor-mio-inositol $^{51,52}$. O 5-desoxi e o 5-desoxi-5-flúor-D-mioinositol $^{53}$ associam-se, respectivamente, 4 e 5 vezes mais facilmente à PI-sintetase que o mio-inositol natural, mas não são transformados pela PI-sintetase no fosfatidilinositol correspondente.

O maior problema encontrado na avaliação biológica destas substâncias é sua dificuldade de penetração através da membrana celular. Parece que este problema é devido à presença de cargas negativas nos grupos fosfatos destas moléculas. Muitos esforços têm sido feitos no sentido de solucionar este problema, como por exemplo a síntese dos "isósteres" mio-inositol 1,4,5-trisulfatos e 1,4,5-trissulfonamidas $^{54}$ ou a proteção dos grupos hidroxila por grupos butiril e $-\mathrm{CH}_{2} \mathrm{CH}\left(\mathrm{CH}_{3}\right)-\left(m-\mathrm{O}_{2} \mathrm{~N}-\mathrm{C}_{6} \mathrm{H}_{4}\right)^{55}$. Infelizmente estes produtos não apresentaram os resultados esperados. Gero e colaboradores ${ }^{16,27,28}$ descreveram a síntese de diferentes inositóis contendo grupos di-butil, di-pentil ou di-hexil fosfatos. Estes derivados apresentaram-se mais ativos devido à sua melhor penetração na célula.

Os conhecimentos sobre o sistema de fosfoinositídeos continuam a progredir rapidamente mas muitos fatores ainda permanecem obscuros, como por exemplo a importância fisiológica da transformação do Ins $(1,4,5) P_{3}$ em diversos compostos fosforilados e a função destes metabólitos intermediários. Poucos análogos dos inositóis polifosforilados sintetizados têm apresentado efeito significativo na cascata dos fosfoinositídeos. Os inositóis polifosforilados são biologicamente importantes mas pouco disponíveis na natureza. Portanto, suas sínteses continuam sendo de fundamental importância para que se possa conhecer melhor a cascata dos fosfoinositídeos e, talvez, possibilitar a descoberta de alguma droga a partir destes conhecimentos. Nos últimos dois anos, inúmeros artigos podem ser encontrados na literatura envolvendo a síntese $\mathrm{s}^{56}$ e/ou os estudos biológicos, bioquímicos, farmacológicos, imunológicos, etc. dos inositóis fosfatos, evidenciando que a pesquisa científica nesta área continua sendo promissora e de grande relevância.

\section{AGRADECIMENTOS}

Os autores M.V. de Almeida e M. V. N. de Souza agradecem ao CNPq e à FAPEMIG por bolsas concedidas. 


\section{REFERÊNCIAS}

1. Cohen, P.; Mouslay, M. D. Em Molecular Mechanisms of Transmembrane Signnaling; Elsevier: Oxford, 1986.

2. Rawls, R. L. Em G Proteins, Research Unravels their Role in Chemical Communication; Chem. Eng. News: Washington, 1986.

3. Michell, R. H.; Trends Biochem. Biophys. Acta 1975, 415, 81.

4. Berridge, M. J.; Dawson, R. M. C.; Downes, C. P.; Heslop, J. P.; Irvine, R. F.; Biochem. J. 1983, 212, 473.

5. Durell, J.; Garlant, J. T.; Cyclitol and Phosphoinositides: Chemistry Metabolism and Function 1968, 165, 743

6. Abdel-Latif, A. A.; Akhtar, R. A.; Smith, J. P.; Ann. N. Y. Acad. Sci. 1978, 121.

7. Reitz, H. L.; ACS Symp. Ser. 1991, 463.

8. Nishizuka, Y.; Nature 1988, 334, 356.

9. Wooten, M. W.; Wrenn, R. W.; FEBS Lett. 1984, 171, 183.

10. Berridge, M. J.; Irvine, R. F.; Nature 1989, 341, 197.

11. Balla, T.; Guillemette, G.; Baukal, R. J.; Catt, K. J.; Biochem. Biophys. Res. Comm. 1987, 148, 199.

12. Szwergold, B. S.; Grahan, R. A.; Brown, T. R.; Biochem. Biophys. Res. Comm. 1987, 149, 874.

13. Irvine, R. F.; Brown, K. D.; Berridge, M. J.; Biochem. J. 1984, 222, 269.

14. Baker, R.; Kulagowski, J. K.; Billington, D. C.; Leeson, P. D.; Lennom, J. C.; Liverton, N.; J. Chem. Soc. Chem. Comm. 1989, 1383.

15. Reitz, H. L.; ACS Symp. Ser. 1991, 563.

16. Almeida, M. V., Tese de Doutorado, Universidade Paris-Sud, Orsay, França, 1993

17. Silva, M. M.; Cleophax, J.; Benício, A. A. A.; Almeida, M. V.; Delaumeny, J. M.; Machado, A. S.; Gero, S. D.; Synlett 1996, 8, 764

18. Dubreuil, D.; Cleophax, J.; Almeida, M. V.; Verre-Sebrié, C.; Liaigre, J.; Vass, G.; Gero, S. D.; Tetrahedron 1997, 53, 16747.

19. Silva, A. D.; Benício, A. A. A.; Gero, S. D.; Tetrahedron Lett. 1999, 40, 6531.

20. Almeida, M. V.; Cleophax, J.; Gateau-Olesker, A.; Prestat, G.; Dubreuil, D.; Gero, S. D.; Tetrahedron 1999, 55, 12997.

21. Benício, A. A. A.; Silva, A. D.; Almeida, M. V.; Silva, M. M.; Gero, S. D.; Tetrahedron 2001, 57, 1161.

22. Almeida, M. V.; Gero, S. D.; Olesker, A. G.; Verre-Sebrié, C.; Dubreuil, D.; Cleophax, J.; Hecker, G.; Wohfeil, S.; Vass, G.; Gao, Z.; Patente Européia A2-0583698, 1994

23. Almeida, M. V.; Bischoff, E.; Olesker, A. G.; Verre-Sebrié, C.; Cleophax, J.; Gero, S. D.; Dubrueil, D.; Vass, G.; Gao, Z.; Wohfeil, S.; Hecker, G.; Patente Européia A1-0583699, 1994.

24. Almeida, M. V.; Cleophax, J.; Dubreuil, D.; Gero, S. D.; Loupy, A. D.; Bioorg. Med. Chem. Lett. 1995, 5, 831.

25. Benício, A. A. A.; Tese de Doutorado, Universidade Federal de Minas Gerais, Brasil, 1996.

26. Machado, A. S.; Tese de Doutorado, Universidade Paris-Sud, Orsay, França, 1985; Machado, A. S.; Dubreuil, D.; Cleophax, J.; Gero, S. D.; Thomas, N. F.; Carbohydr. Res. 1992, 233.

27. Almeida, M. V.; Dubreuil, D.; Cleophax, J.; Verre-Sebrié, C.; Pipelier, M.; Presta, G.; Vass, G.; Gero, S. D.; Tetrahedron 1999, 55, 7251.

28. Dubreuil, D.; Cleophax, J.; Almeida, M. V.; Verre-Sebrié, C.; Vass, G.; Gero, S. D.; Tetrahedron 1999, 55, 7573.

29. Ferrier, R. J.; J. Chem. Soc. Perkin Trans. I 1979, 1433.

30. Ferrier, R. J.; Blattner, R.; Haines, S. R.; J. Chem. Soc., Perkin Trans. I 1985, 2413.

31. Ferrier, R. J.; Blattner, R.; J. Chem. Soc., Chem. Comm. 1987, 1008

32. Watanabe Y.; Ogasawara T.; Nakahira H.; Matsukli T.; Ozaki S.; Tetrahedron Lett. 1988, 41, 5259.

33. Vacca, J. P.; De Solms, S. J.; Huff, J. R.; J. Am. Chem. Soc. 1987, 109, 3478 .
34. Meek J. L.; Da Vidson, F.; Hobbs F. W.; J. Am. Chem. Soc. 1988, 110, 2317.

35. Bruzik, K. S.; Salamonczyk, G. M.; Carbohydr. Res. 1989, 195, 67.

36. Falck, J. R.; Yadagiri, P.; J. Org. Chem. 1989, 54, 5851; Lesuisse, D.; Berchtold, G. A.; J. Org. Chem. 1985, 50, 888

37. Kozikowski, A. P.; Ognyanov, V. S.; Fauq, A. H.; Wilcox, R. H.; Nahorski, S. R.; J. Chem. Soc., Chem. Comm. 1994, 599.

38. Ley, S. V.; Parra, M.; Redgrave, A. J; Sternfeld, F.; Vidal, A.; Tetrahedron Lett. 1989, 30, 3557; Ley, S. V.; Sternfeld, F.; Tetrahedron Lett. 1988, 29 , 5305.

39. Spiers, I. D.; Freeman, S.; Poyner, D. R.; Schawalbe, C. H.; Tetrahedron Lett. 1995, 36, 2125.

40. Hambin, M. R.; Potter, B. V. L.; Gigg, R.; J. Chem. Soc., Chem. Comm. 1987, 628

41. Taylor, C. W.; Berridge, M. J.; Brown, K. D.; Cooke, A. M.; Potter, B. V. L.; Biochem. Biophys. Res. Comm. 1988, 150, 626.

42. Delisle, S.; Krause, K. H.; Denning, G.; Potter, B. V. L.; Welsh, M. J.; J. Biol. Chem. 1990, 265, 11726.

43. Strupish, J.; Cooke, A. M.; Potter, B. V. L.; Gigg, R.; Nahorski, S. R.; Biochem. J. 1988, 253, 901.

44. Willicolks, A. L.; Potter, B. V. L.; Cooke, A. M.; Nahorski, S. R.; Eur. J. Pharmacol. 1988, 155, 181.

45. Taylor, C. W.; Berridge, M. J.; Cooke, A. M.; Potter, B. V. L.; Biochem. J. 1989, 259, 645.

46. Cooke, A. M.; Nahorski, S. R.; Potter, B. V. L.; FEBS Lett. 1989, 243, 373.

47. Westerduin, P.; Van Boeckel, C. A. A.; ACS Symp. Ser. 1991.

48. McLaurin, J.; Golomb, R.; Jurewicz, A.; Antel, J. P.; Fraser, P. E.; J. Biol. Chem. 2000, 275, 18495

49. Shimohama, S.; Tanino, H.; Sumida, Y.; Tsuda, J.; Fujimoto, S.; Neuroscience Lett. 1998, 245, 159.

50. Huang, W.; Alexander, G. E.; Daly, E. M.; Shetty, H. U.; Krasuski, J. S.; Rapoport, S. I.; Schapiro, M. B.; Am. J. Psychiatry 1999, 156, 1879.

51. Kozikowski, A. P.; Powis, G.; Fauq, A. H.; Tuckmantel, W.; Gallegos, A.; J. Org. Chem. 1994, 59, 963.

52. Powis, G.; Aksoy, I. A; Melder,D. C.; Aksoy, S.; Eichinger, H.; Fauq, A. H., Kozikowski, P.; Cancer Chemother. Pharmacol. 1991, 29, 95.

53. Moyer, J. D.; Reizes, O; Ahir, S.; Jiang, C.; Malinowski, N.; Baker, D. C.; Mol. Pharmacol. 1988, 33, 683.

54. Westerduin, P.; Willens, A. M.; Van Boeckel, C. A. A.; Tetrahedron Lett. 1990, 31, 6919.

55. Tegge, W., Tese de Doutorado, Universidade de Bremen, França, 1986.

56. Hosoda, A.; Nomura, E.; Murakami, A.; Koshimizu, K.; Ohigashi, H.; Mizuno, K.; Taniguchi, H.; Bioorg. Med. Chem. 2002, 10, 1855; Jardine, M. A.; Spies, H. S. C.; Nkambule, C. M.; Gammon, D. W.; Steenkamp, D. J.; Bioorg. Med. Chem. 2002, 10, 875; Ravikumar, K. S.; Farquhar, D.; Tetrahedron Lett. 2002, 43, 1367; Lindberg, J.; Ohberg, L.; Garegg, P. J.; Tetrahedron 2002, 58, 1387; Khersonsky, S. M.; Chang, Y. T.; Carbohydr. Res. 2002, 337, 75; Nishikawa, A.; Saito, S.; Hashimoto, Y.; Koga, K.; Shirai, R.; Tetrahedron Lett. 2001, 42, 9195; Wang, S.; Chen, C.-S.; Bioorg Med. Chem. 2001, 9, 3165; Adelt, S.; Plettenburg, O.; Dallmann, G.; Ritter F. P.; Shears, S. B.; Altenbach, H. J.; Vogel, G.; Bioorg. Med. Chem. Lett. 2001, 11, 2705; Hosoda, A.; Nomura, E.; Mizuno, K.; Taniguchi, H.; J. Org. Chem. 2001, 66, 7199; Scumlimbrene, B. R.; Miller, S. J.; J. Am. Chem. Soc. 2001, 123, 10125;Liu, C.; Riley, A. M.;Yang, X.; Shears, S B.; Potter, B. V.; J. Med. Chem. 2001, 44, 2984; Jaworek, C. H.; Iacobucci, S.; Calias, P.; d'Alarcao M., Carbohydr. Res. 2001, 331, 375; Takahashi, H.; Kittaka, H.; Ikegami, S. J. Org. Chem. 2001, 66, 2705; Schnaars, A Schultz, C.; Tetrahedron 2001, 57, 519; Horne, G.; Potter, B. V.; Chemistry 2001, 7, 80; Wang, D. S.; Hsu, A. L.; Chen, C. S.; Bioorg. Med. Chem. 2001, 9, 133; Hu, Y.; Meuillet, E. J.; Berggren M.; Powis, G.; Kozikowski, A. P.; Bioorg. Med. Chem. Lett. 2001, 11, 173 . 